\title{
Free Vibrations of Multilayer Anisotropic Cylindrical Shells
}

\author{
C. W. BERT \\ J. L. BAKER * \\ - School of Aerospace and Mechanical Engineering \\ University of Oklahoma, Norman, Oklahoma 73069
}

D. M. EgLE

(Received May 10, 1969)

\begin{abstract}
A theoretical analysis is presented for determining the free vibrational characteristics of thin-walled, circular cylindrical shells with layers of anisotropic elastic material arbitrarily laminated either symmetrically or unsymmetrically about the shell middle surface. An arbitrarily laminated, anisotropic version of Love's first-approximation shell theory is used to formulate the coupled equations of motion. An exact solution with a classical checkerboard nodal pattern is found for the case of a shell with specially orthotropic layers arbitrarily laminated and with freely supported ends. For a boron/epoxy composite cylinder, the significant effect of omitting bending-stretching coupling is demonstrated and various lamination arrangements are investigated. Also, a general solution is presented for the axisymmetric modes of an arbitrarily laminated anisotropic shell. Finally, an approximate solution, using a combination of two helical-nodal-pattern modes, is obtained for the unsymmetric modes of the same general class of shell with a supported boundary condition.
\end{abstract}

\section{INTRODUCTION}

AMINATED FILAMENTARY composite materials are becoming of rapidly 1 increasing importance in aerospace and hydrospace applications. The cylindrical shell configuration is widely used in launch-vehicle structures, reentry vehicles, aircraft fuselages, and hulls of submersibles. Thus, there is considerable current interest in the vibrational characteristics of cylindrical shells constructed of laminated filamentary composite materials.

A single layer of a filamentary composite material behaves macroscopically as if it were a homogeneous orthotropic material, i.e. one

\footnotetext{
'Presented at the Fourth Annual Symposium on High Performance Composites, Washington University, St. Louis, Mo., April 8-9, 1969.

-Also Structures Engineer, D. Long Engineering, Inc., Norman, Oklahoma.
} 
having three mutually perpendicular planes of material symmetry. If the material-symmetry axes are lined up with the shell-surface principal coordinates (i.e. the axial and circumferential directions), the shell is said to be specially orthotropic. Since cylindrical shells with closely spaced ring and/or stringer stiffeners also can be approximated by considering them to be specially orthotropic, a number of analyses have been carried out for such shells, for example [1,2].

If the material-symmetry axes are not lined up with the shell principal coordinates, the shell is said to be anisotropic (or generally orthotropic). This induces coupling between the membrane and in-surface shear effects and between bending and twisting effects. Since there is no structural advantage, or even requirement, for shells constructed in this way, apparently it has not been subjected to analysis.

When a shell is constructed of more than one isotropic layer with each layer having different elastic properties and the layers arranged symmetrically with respect to the shell middle surface, the governing equations are identical to those of a single-layer isotropic shell [3]. However, if the isotropic layers are arranged unsymmetrically with respect to the shell middle surface, there is coupling between in-surface (stretching and/or shear) and out-of-plane (bending and/or twisting) effects. This is called bending-stretching coupling and it is greatest for two-layer shells [4] such as those used in reentry-vehicle heat shields.

For a shell constructed of multiple orthotropic layers, with the layers aligned either axially or circumferentially and arranged symmetrically with respect to the shell middle surface, the equations are the same as those for a single-layer specially orthotropic shell [5]. If the specially orthotropic layers are arranged unsymmetrically, bending-stretching coupling is induced. Such shells were considered in [5] but only approximately in terms of an equivalent single-layer specially orthotropic shell. Using a Donnell-type shell theory, Dong [7] considered arbitrarily laminated, specially orthotropic cylindrical shells of finite length.

The most general case of laminated shells is that of arbitrarily laminated anisotropic layers, i.e. multiple orthotropic layers arbitrarily oriented and arbitrarily arranged. Kunukkasseril [8] analyzed simple helical-mode extensional vibrations for an infinitely long shell of this type. Also an approximate inextensional solution was obtained for a finitelength shell. The present analysis is believed to be the first to consider general vibrational modes of finite-length shells with arbitrarily laminated anisotropic layers.

\section{HYPOTHESES}

The following hypotheses form the bases for the analysis: 
H1. Displacements are small compared to the shell thickness, so that the strain-displacement relations may be assumed to be linear.

H2. The Kirchhoff hypothesis is applicable, i.e. line elements normal to the middle surface before deformation remain straight, normal to the deformed middle surface, and unchanged in length after deformation.

H3. The ratio of the shell thickness to the radius of the middle surface is small as compared with unity, i.e. Love's first-approximation shell theory [9] is used.

H4. All components of translational inertia are included, but all components of rotatory inertia, as well as all dissipative effects, are neglected.

H5. Each individual layer is considered to behave macroscopically as a homogeneous, anisotropic, linearly elastic material.

H6. Each layer may be of arbitrary thickness (within the limitation of H3), properties, and may be arranged either symmetrically or unsymmetrically with respect to the middle surface.

H7. The layers are assumed to be bonded together with a perfect bond (massless, infinitesimal thickness, no relative deformation at the interface).

Hypotheses $\mathrm{HI}-\mathrm{H} 4$ are the bases for small-displacement, thin elastic shell dynamics. It is emphasized that the simplifying assumptions of the Donnell type [10] are not used, although the more exact strain-displacement expressions of the Love second-approximation [9] or Flügge exact theory [11] are not employed either. Hypothesis $\mathrm{H} 4$ is consistent with the neglect of transverse shear flexibility (H2).

\section{FORMULATION OF EQUATIONS OF MOTION}

The differential equations of motion are formulated in terms of the three middle-surface displacement components $u, v, w$.

In view of hypotheses $\mathrm{H} 1-\mathrm{H} 3$, the linear equations relating the middlesurface strain components to the displacements are as follows:

$$
\bar{\epsilon}_{1}=u_{, x} ; \quad \bar{\epsilon}_{2}=v_{, y}+R^{-1} w ; \quad \bar{\epsilon}_{6}=u_{, y}+v_{, x}
$$

where a subscript comma denotes differentiation with respect to the variable following the comma. The curvature components are related to the displacements by the following linear equations:

$$
\kappa_{1}=-w_{, x x} ; \quad \kappa_{2}=-w_{, y y}+R^{-1} v_{, y} ; \quad \kappa_{6}=-2 w_{, x y}+R^{-1} v_{, x}
$$

The total strains at an arbitrary distance $z$ from the middle surface are:

$$
\epsilon_{j}=\bar{\epsilon}_{j}+\kappa_{j} z ; \quad j=1,2,6
$$


In view of $\mathbf{H} 3, \mathrm{H} 5$, and $\mathrm{H} 6$, each individual layer is assumed to be in a state of generalized plane stress governed by the two-dimensional Hooke's law:

$$
\left\{\sigma_{i}\right\}=\left[Q_{i j}\right]\left\{\epsilon_{j}\right\} ; \quad i, j=1,2,6
$$

In the general anisotropic case, the $Q_{i j}$ matrix is fully populated.

Using Love's first-approximation shell theory, the stress resultants and stress couples are defined as follows:

$$
N_{i}=\int_{-h / 2}^{h / 2} \sigma_{i} d z ; \quad M_{i}=\int_{-h / 2}^{h / 2} z \sigma_{i} d z
$$

Putting (4) into (5) yields the following constitutive relations for the composite shell:

$$
\left\{\begin{array}{l}
N_{i} \\
M_{i}
\end{array}\right\}=\left[\begin{array}{ll}
A_{i j} & B_{i j} \\
B_{i j} & D_{i j}
\end{array}\right]\left\{\begin{array}{l}
\bar{\epsilon}_{j} \\
\kappa_{j}
\end{array}\right\} ; \quad i, j=1,2,6
$$

where the stretching, bending-stretching coupling, and bending stiffness submatrices are defined as follows:

$$
\left(A_{i j}, B_{i j}, D_{i j}\right)=\int_{-h / 2}^{h / 2}\left(1, z, z^{2}\right) Q_{i j} d z
$$

For a shell laminated symmetrically with respect to the middle surface, the bending-stretching submatrix $B_{i j}$ vanishes.

Considering H4, Newton's second law can be applied to a differential element of the shell to obtain the following three equations of translational motion and two equations of rotational equilibrium:

$$
\begin{aligned}
& N_{1, x}+N_{6, y}=\bar{M} u_{, t t} \\
& N_{2, y}+N_{6, x}+R^{-1} Q_{2}=\bar{M} v_{, t t} \\
& Q_{1, x}+Q_{2, y}-R^{-1} N_{2}=\bar{M} w_{, t t} \\
& M_{1, x}+M_{6, y}-Q_{1}=0 \\
& M_{2, y}+M_{6, x}-Q_{2}=0 \\
& \text { where } \\
& \bar{M}=\int_{-h / 2}^{h / 2} \rho d z
\end{aligned}
$$

Equations (11) and (12) can be used to eliminate the transverse shear resultants from equations (9) and (10) with the following results:

$$
\begin{aligned}
& N_{2, y}+N_{6, x}+R^{-1}\left(M_{2, y}+M_{6, x}\right)=\bar{M} v_{, t t} \\
& M_{1, x x}+2 M_{6, x y}+M_{2, y y}-R^{-1} N_{2}=\bar{M} w_{, t t}
\end{aligned}
$$

Substituting equations (6) into motion equations (8), (13), and (14) 
yields the following set of coupled equations in terms of the displacements $u, v, w$ :

$$
\begin{aligned}
& \left(A_{11} u_{, x x}+2 A_{16} u_{, x y}+A_{66} u_{, y y}-\bar{M} u_{, t t}\right)+\left(\bar{A}_{16} v_{, x x}+\bar{A}_{12} v_{, x y}+\bar{A}_{26} v_{, y y}\right) \\
& +\left(A_{12}^{*} w_{, x}+A_{26}^{*} w_{, y}-B_{11} w_{, x x x}-3 B_{16} w_{, x x y}-B_{17} w_{, x y y}-B_{26} w_{, y y y}\right)=0 \\
& \left(\bar{A}_{16} u_{, x x}+\bar{A}_{12} u_{, x y}+\bar{A}_{26} u_{, y y}\right)+\left(A_{66}^{\prime} v_{, x x}+2 A_{26}^{\prime} v_{, x y}+A_{22}^{\prime} v_{, y y}\right. \\
& \left.-\bar{M} v_{, t t}\right)+\left(\bar{A}_{26}^{*} w_{, x}+\bar{A}_{22}^{\circ} w_{, y}-\bar{B}_{16} w_{, x x x}-\bar{B}_{17} w_{, x x y}-3 \bar{B}_{26} w_{, x y y}\right. \\
& \left.-\bar{B}_{22} w_{, y y y}\right)=0 \\
& \left(A_{12}^{*} u_{, x}+A_{26}^{*} u_{, y}-B_{11} u_{, x x x}-3 B_{16} u_{, x x y}-B_{17} u_{, x y y}-B_{26} u_{, y y y}\right) \\
& +\left(\bar{A}_{26}^{*} v_{, x}+\bar{A}_{22}^{*} v_{, y}-\bar{B}_{16} v_{, x x x}-\bar{B}_{17} v_{, x x y}-3 \bar{B}_{26} v_{, x y y}-\bar{B}_{22} v_{, y y y}\right) \\
& +\left(A_{22}^{*} R^{-1} w-2 B_{12}^{*} w_{, x x}-4 B_{26}^{*} w_{, x y}-2 B_{22}^{*} w_{, y y}+D_{11} w_{, x x x x}+4 D_{16} w_{, x x x y}\right. \\
& \left.+2 D_{17} w_{, x x y y}+4 D_{26} w_{, x y y y}+D_{22} w_{, y y y y}+\bar{M}_{, t t}\right)=0
\end{aligned}
$$

where

$$
\begin{array}{lc}
\bar{A}_{12}=A_{12}+B_{12} R^{-1}+A_{66}+B_{66} R^{-1} & \\
\bar{A}_{i j}=A_{i j}+B_{i j} R^{-1} & (i j=16,22,26) \\
B_{17}=B_{12}+2 B_{66} ; & D_{17}=D_{12}+2 D_{66} \\
\bar{B}_{i j}=B_{i j}+D_{i j} R^{-1} & \left({ }_{i j}=16,17,22,26\right) \\
A_{i j}^{\prime}=\bar{A}_{i j}+\bar{B}_{i j} R^{-1} & (i j=22,26,66) \\
\left(A_{i j}^{*}, B_{i j}^{*}\right)=\left(A_{i j}, B_{i j}\right) R^{-1} &
\end{array}
$$

Equations (15) can be written in abbreviated linear differential operator form as follows:

$$
\left[\begin{array}{lll}
L_{1} & L_{2} & L_{3} \\
L_{2} & L_{4} & L_{5} \\
L_{3} & L_{5} & L_{6}
\end{array}\right]\left\{\begin{array}{c}
u \\
v \\
w
\end{array}\right\}=0
$$

where

$$
\begin{aligned}
L_{1} & =A_{11} d_{x}^{2}+2 A_{16} d_{x} d_{y}+A_{66} d_{y}^{2}-\bar{M} d_{t}^{2} \\
L_{22} & =\bar{A}_{16} d_{x}^{2}+\bar{A}_{12} d_{x} d_{y}+\bar{A}_{26} d_{y}^{2} \\
L_{3} & =A_{16}^{*} d_{x}+A_{26}^{*} d_{y}-B_{11} d_{x}^{3}-3 B_{16} d_{x}^{2} d_{y}-B_{17} d_{x} d_{y}^{2}-B_{26} d_{y}^{3} \\
L_{4} & =A_{66}^{\prime} d_{x}^{2}+2 A_{26}^{\prime} d_{x} d_{y}+A_{22}^{\prime} d_{y}^{2}-\bar{M} d_{t}^{2} \\
L_{5} & =\bar{A}_{26}^{*} d_{x}+\bar{A}_{22}^{\star} d_{y}-\bar{B}_{16} d_{x}^{3}-\bar{B}_{17} d_{x}^{2} d_{y}-3 \bar{B}_{26} d_{x} d_{y}^{2}-\bar{B}_{22} d_{y}^{3} \\
L_{6} & =\bar{A}_{22}^{*} R^{-1}-2 B_{12}^{*} d_{x}^{2}-4 B_{26}^{*} d_{x} d_{y}-2 B_{22}^{*} d_{y}^{2}+D_{11} d_{x}^{4}+4 D_{16} d_{x}^{3} d_{y} \\
& +2 D_{17} d_{x}^{2} d_{y}^{2}+4 D_{26} d_{x} d_{y}^{3}+D_{22} d_{y}^{4}+\bar{M} d_{t}^{2}
\end{aligned}
$$


Using the procedure originated by Batdorf [12] in 1947, being careful not to take any derivatives, equations (17) can be combined into the following single integrodifferential equation:

$$
\left[L_{3}\left(\frac{L_{4}^{-1} L_{5}-L_{2}^{-1} L_{3}}{L_{2}^{-1} L_{1}-L_{4}^{-1} L_{2}}\right)+L_{5}\left(\frac{L_{2}^{-1} L_{5}-L_{1}^{-1} L_{3}}{L_{1}^{-1} L_{2}-L_{2}^{-1} L_{4}}\right)+L_{6}\right](w)=0
$$

where $L_{i}^{-1}$ denotes the integral operator which is the inverse of differential operator $L_{i}$.

As discussed in a very recent paper by Batdorf [13], an integrodifferential equation of the above type cannot be differentiated to convert it to a differential equation without risking the introduction of unwanted solutions. If equation (19) is escalated in this manner, the following differential is obtained.

$$
\left[\left(L_{2}^{2}-L_{1} L_{4}\right) L_{6}+L_{3}\left(L_{3} L_{4}-L_{2} L_{5}\right)+L_{5}\left(L_{1} L_{5}-L_{2} L_{3}\right)\right](w)=0
$$

Equation (20) can be obtained also by setting the determinant of the operator matrix in equation (17) equal to zero. Equation (20) agrees with the result obtained by Nikulin [14] by another method. However, the definitions of the operators $L_{i}$ used here for the laminated anisotropic case are considerably more complicated than in the homogeneous isotropic case considered by Nikulin.

In principle, an exact solution of the problem could be obtained by assuming a solution in the following form:

$$
w=\sum_{k=1}^{8} W_{k} e^{i\left(\lambda_{k} x+\beta_{n} y+\omega t\right)}
$$

where $W_{k}, \lambda_{k}, \beta_{n}$, and $\omega$ are constants. This would lead to a characteristic equation in the form of an eighth-order polynomial in $\lambda_{k}$ as a function of $\beta_{n}$ and $\omega$. For homogeneous, isotropic cylindrical shells, such a method of solution was suggested by Flügge in 1934 [11] and carried out by Forsberg [15] for the isotropic case and by Dong [7] for the laminated specially orthotropic case. For these cases, only even partial derivatives appear and the roots of the characteristic equations are of the form:

$$
\lambda= \pm a, \pm b, \pm(c \pm d)
$$

where $a, b, c, d$ are real quantities. However, in the laminated anisotropic case considered here, not only do various odd partial derivatives appear, but also the algebra is highly complicated.

\section{EXACT SOLUTION FOR LAMINATED SPECIALLY ORTHOTROPIC SHELL WITH BOTH ENDS FREELY SUPPORTED}

One of the boundary conditions treated most often in the literature is 
C. W. Bert, J. L. Baker and D. M. Egle
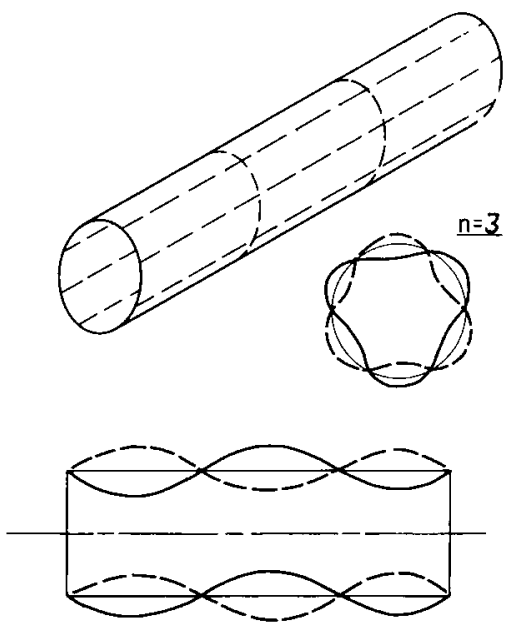

$\underline{m=3}$

Figure 1. Checkerboard nodal patterns.

Table 1. Comparisons Among Theoretical and Experimental Resonant Frequencies (cps) for a Homogeneous, Isotropic Cylinder with Freely Supported Ends

\begin{tabular}{|c|c|c|c|c|c|c|}
\hline \multirow[b]{2}{*}{$n$} & \multicolumn{3}{|c|}{$m=1$} & \multicolumn{3}{|c|}{$m=2$} \\
\hline & a & b & c & $a$ & b & c \\
\hline 1 & 3270.5 & 3271.0 & - & 4837.7 & 4837.9 & - \\
\hline 2 & 1862.0 & 1862.3 & - & 3725.0 & 3725.5 & - \\
\hline 3 & 1101.8 & 1102.0 & 1001 & 2742.6 & 2743.1 & - \\
\hline 4 & 705.7 & 705.9 & 715. & 2018.0 & 2018.5 & - \\
\hline 5 & 497.5 & 497.7 & 534. & 1515.0 & 1515.4 & 1527. \\
\hline 6 & 400.1 & 400.3 & 410. & 1174.9 & 1175.2 & - \\
\hline 7 & 380.7 & 380.9 & 393. & 953.6 & 953.9 & - \\
\hline 8 & 416.7 & 416.8 & 426. & 824.2 & 824.6 & - \\
\hline 9 & 488.6 & 488.7 & 495. & 770.4 & 770.6 & 744. \\
\hline 10 & 583.9 & 583.9 & 587. & 778.3 & 778.6 & 782. \\
\hline 11 & 696.3 & 696.3 & 697. & 834.2 & 834.4 & 834. \\
\hline 12 & 822.7 & 822.8 & 821. & 925.5 & 925.7 & 927. \\
\hline 13 & 961.9 & 961.9 & 965. & 1043.0 & 1043.1 & 1039. \\
\hline 14 & 1113.2 & 1113.2 & 1116 & 1180.7 & 1180.9 & 1180. \\
\hline 15 & 1276.2 & 1276.2 & 1281 & 1335.2 & 1335.3 & 1333. \\
\hline 16 & 1450.7 & 1450.7 & 1454 & 1504.1 & 1504.2 & 1499. \\
\hline 17 & 1636.6 & 1636.6 & 1636. & 1686.4 & 1686.5 & 1680. \\
\hline 18 & 1833.9 & 1833.9 & 1832. & 1881.3 & 1818.4 & 1879. \\
\hline 19 & 2042.6 & 2042.6 & 2047 & 2088.3 & 2088.3 & 2086. \\
\hline \multicolumn{7}{|c|}{$\begin{array}{l}\text { Material properties: Young's modulus, } 29.5 \times 10^{\text {an }} \text { psi; Poisson's ratio, } 0.285 \text {; density, } 734 \times 10^{-6} \text { lb-sec } / \text { in. }^{4} \\
\text { Shell dimensions: } h=0.02 \text { in.; } L=11.74 \text { in., } R=5.836 \text { in. } \\
\text { aFlügge shell theory, Ref. } 17 \text {. } \\
\text { opresent theory. } \\
\text { 'Experimental results, Ref. } 16 \text {. }\end{array}$} \\
\hline
\end{tabular}


that of simple support without axial restraint (often called freely supported):

$$
v=w=N_{1}=M_{1}=0
$$

It can be shown that for a shell with these boundary conditions at each end, the following set of functions satisfy both the differential equations (17) and the boundary conditions, thus constituting an exact solution:

$$
\begin{gathered}
u=U \cos \alpha_{m} x \cos \beta y \sin \omega t, v=V \sin \alpha_{m} x \sin \beta y \sin \omega t, \\
w=W \sin \alpha_{m} x \cos \beta y \sin \omega t
\end{gathered}
$$

where $\alpha_{m}=m \pi / L, \beta=n / R$. The modal shape represented by the expression for $w$ in (23) is often referred to as a checkerboard type, since it has nodes consisting of axial and circumferential lines forming a checkerboard pattern (see Figure 1).

Since no published results of vibrational experiments on unsymmetrically laminated specially orthotropic cylindrical shells are known to the authors, a complete check on the analysis by comparison with experimental data could not be made. Furthermore, it takes painstaking care in the experimental setup to achieve physically the freely supported boundary condition. The present analysis was applied to the case of a homogeneous isotropic cylinder investigated experimentally by Egle and Bray [16] and theoretically using an exact Flügge type solution due to Egle and Soder [17]. The agreement among all three results was quite good as shown in Table 1 . This not only verifies the applicability of the Love first-approximation shell theory used in the present paper, but also it serves as a check on the algebra and programming.

Although the end conditions used in the experiments of Weingarten [3] were those of an elastic support, he carried out a series of calculations for a homogeneous, isotropic cylinder with freely supported ends. His analysis utilized Donnell's shell theory. The results of his calculations are compared with those of the present analysis, which uses Love's firstapproximation theory, as shown in Table 2 . The good agreement at higher circumferential wave numbers indicates that in this region, the more approximate Donnell theory is probably adequate.

Weingarten also showed that laminated shells consisting of isotropic layers all having the same Poisson's ratio are mechanically equivalent to a homogeneous, isotropic shell. The results of his Donnell-theory analysis of a symmetrically laminated, three-layer shell, using this equivalent homogeneous shell hypothesis, are listed in Table 3 , along with the results of the present analysis. It is seen that the differences between the two analyses are even less than those for the homogeneous shell of Table 2. This corroborates the equivalent shell hypothesis. 
Table 2. Comparison Between Resonant Frequencies Calculated by Donnell and Love First-Approximation Shell Theories for a Homogeneous, Isotropic Cylinder with Freely Supported Ends

\begin{tabular}{|c|c|c|c|}
\hline \multirow[b]{2}{*}{$\begin{array}{l}\text { No. of Axial } \\
\text { Half Waves, } m\end{array}$} & \multirow[b]{2}{*}{$\begin{array}{l}\text { No. of Circum- } \\
\text { ferential Waves, } n\end{array}$} & \multicolumn{2}{|c|}{ Resonant Frequency, cps } \\
\hline & & $\begin{array}{l}\text { Donnell-Type } \\
\text { Analysis [3] }\end{array}$ & $\begin{array}{l}\text { Present } \\
\text { Analysis }\end{array}$ \\
\hline \multirow[t]{15}{*}{1} & 1 & 4496 & 4332 \\
\hline & 3 & 1449 & 1397 \\
\hline & 4 & 917 & 884 \\
\hline & 5 & 635 & 613 \\
\hline & 6 & 494 & 476 \\
\hline & 7 & 446 & 431 \\
\hline & 8 & 467 & 451 \\
\hline & 9 & 534 & 515 \\
\hline & 10 & 631 & 609 \\
\hline & 11 & 749 & 722 \\
\hline & 12 & 883 & 852 \\
\hline & 13 & 1032 & 995 \\
\hline & 14 & 1194 & 1150 \\
\hline & 15 & 1369 & 1318 \\
\hline & 16 & 1556 & 1499 \\
\hline \multirow[t]{9}{*}{2} & 5 & 2006 & 1933 \\
\hline & 6 & 1534 & 1479 \\
\hline & 8 & 1028 & 991 \\
\hline & 9 & 926 & 893 \\
\hline & 12 & 1017 & 981 \\
\hline & 15 & 1435 & 1382 \\
\hline & 17 & 1807 & 1742 \\
\hline & 18 & 2015 & 1942 \\
\hline & 19 & 2237 & 2155 \\
\hline \multirow[t]{3}{*}{3} & 9 & 1637 & 1578 \\
\hline & 11 & 1377 & 1327 \\
\hline & 24 & 3606 & 3474 \\
\hline \multirow{3}{*}{4} & 9 & 2458 & 2369 \\
\hline & 11 & 2181 & 1914 \\
\hline & 16 & 1984 & 1912 \\
\hline $\begin{array}{l}\text { Material properties: You } \\
\text { Shell dimensions: } \mathrm{h}=0\end{array}$ & $\begin{array}{l}\text { lus, } 30.0 \times 10^{6} \mathrm{psi} ; \text { Pois } \\
8.60 \text { in.; R } \\
8.00 \text { in. }\end{array}$ & tio, 0.30 & $\sec ^{2} /$ in. $^{4}$ \\
\hline
\end{tabular}

Using a Donnell-type shell theory, Dong [7] carried out frequency calculations for a two-layer cylinder with freely supported ends. The elastic coefficients, $Q_{i j}$, when converted to the usual engineering moduli, are as follows:

Major Young's modulus, psi

Minor Young's modulus, psi

Major Poisson's ratio

Shear modulus, psi

Density

Layer orientation

Layer thickness, in.
Inner Layer

$29.3 \times 10^{6}$

$29.3 \times 10^{6}$

0.333

$13.2 \times 10^{6}$

$\rho$

Axial or Circumferential

0.20
Outer Layer

$9.87 \times 10^{6}$

$5.50 \times 10^{6}$

0.565

$2.51 \times 10^{6}$

$0.5 \rho$

Circumferential

0.20 
Table 3. Comparison Between Resonant Frequencies Calculated in [3] and the Present Analysis for a Three-Layer, Isotropic Cylinder with Freely Supported Ends

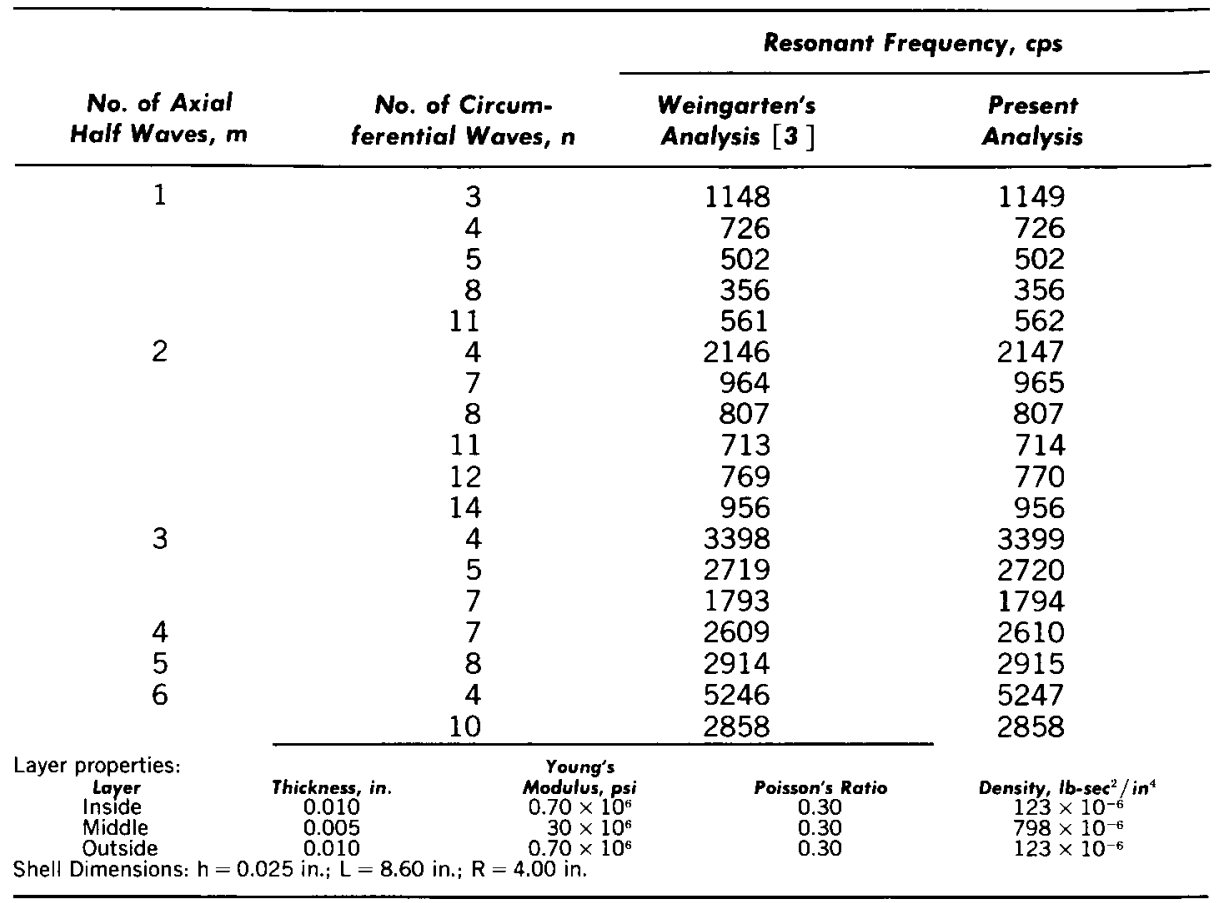

It is noted that both layers are orthotropic. Dong intended the inner layer to be isotropic. However, although the major and minor Young's moduli of the inner layer are equal, its shear modulus is not $1 /[2(1+$ Poisson's ratio $)]$ times the Young's modulus and thus it is not orthotropic.

Only relative values, rather than numerical values, were given for the layer densities, since the frequency results were presented in dimensionless form, normalized by the lowest extensional ring frequency given by:

$$
\omega_{0}=R^{-1}\left(A_{22} / \bar{M}\right)^{1 / 2}
$$

The shell dimensions used were: $h=0.4 \mathrm{in} ., L=200 \mathrm{in}$., $R=10 \mathrm{in}$.

It is noted that since the example used by Dong was a two-layered one, bending-stretching coupling was present. Figure 2 presents curves depicting natural frequencies associated with four different axial half-wave numbers as a function of circumferential wave number, as determined by Dong's Donnell-type analysis as well as the present analysis using Love's first-approximation theory. 


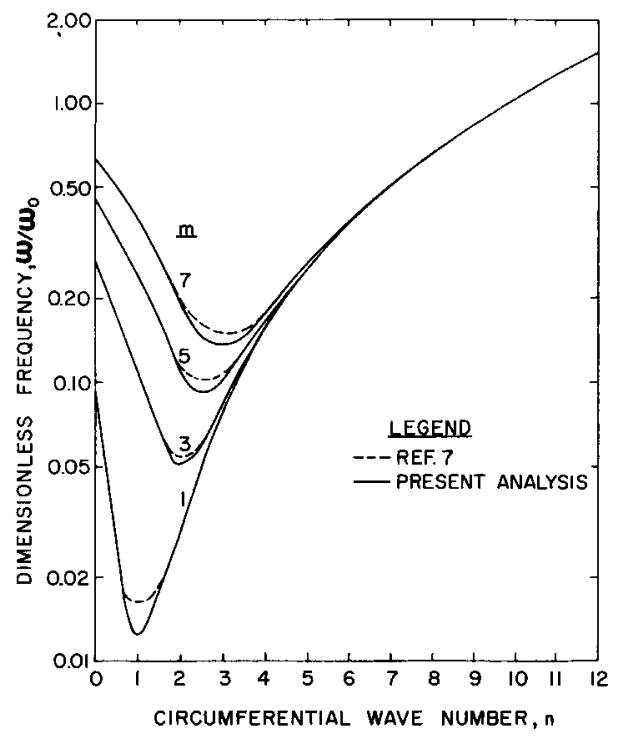

Figure 2. Dimensionless frequency versus circumferential wave number for a twolayer specially-orthotropic cylinder as computed by Dong and the present analysis.

The bending-stretching coupling effect is greatest for the case of two layers cross plied of a highly orthotropic material. It is noted that the materials used in Dong's example are not as highly orthotropic as boron/epoxy which is of considerable interest in airframe applications. Therefore, a two-layer, cross-ply cylinder having the following properties, typical of boron/epoxy [18] was studied:

Major Young's modulus, $31.0 \times 10^{6} \mathrm{psi}$ Major Poisson's ratio, 0.28

Minor Young's modulus, $2.7 \times 10^{6}$ psi Shear modulus, $0.75 \times 10^{6}$ psi

Density, $192 \times 10^{-6} \mathrm{lb}-\mathrm{sec}^{2} / \mathrm{in}^{4}$

The shell dimensions used were: $h=0.02$ in., $L=31.5 \mathrm{in} ., R=2.481 \mathrm{in}$.

The results are shown by the solid lines in Figure 3 as a function of circumferential wave number $n$. For comparison, the dashed lines denote the frequencies calculated by omitting the bending-stretching terms (i.e., by setting $B_{i j}=0$ ). It can be seen that the effect of omitting the coupling was negligible in the lower- $n$ modes; however, at higher values of $n$, omission of coupling resulted in erroneous frequencies appreciably higher than the correct values obtained by including coupling.

For design guidance, a series of calculations was made to study the effects of various three-ply, specially orthotropic, lamination arrangements of boron/epoxy on the lowest frequency. The parameters are the same as 
Free Vibrations of Multilayer Anisotropic Cylindrical Shells

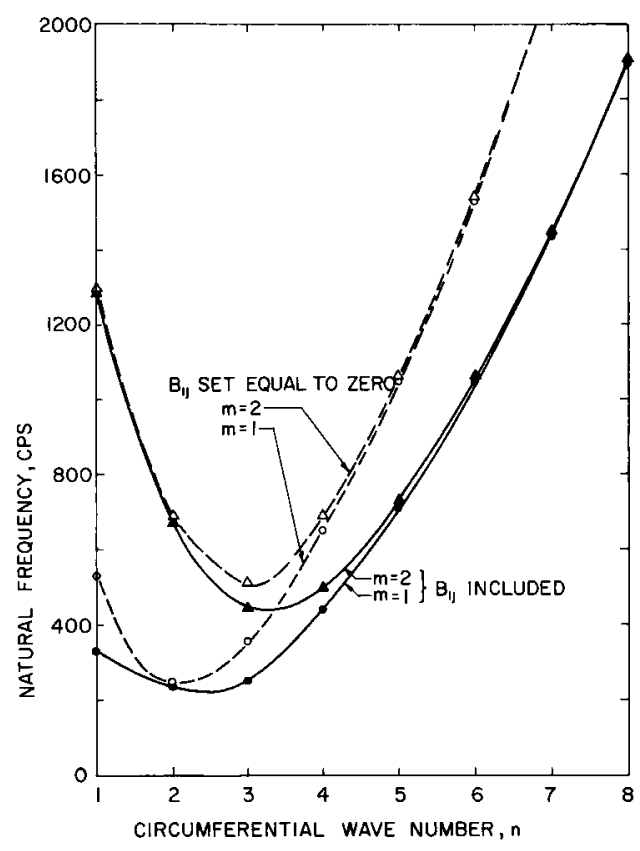

Figure 3. Effect of bending-stretching coupling ( $B_{i j}$ terms) on natural frequencies for a two-layer, cross-ply boron/epoxy cylinder.

the two-ply cylinder mentioned above, except here $h=0.03 \mathrm{in}$. The results are presented in Figure 4 . It is noted that the natural frequencies for one axial and two circumferential plies nearly coincide with those for the all-circumferential case, except at $n=1$ and 2 . Similarly the frequencies for one circumferential and two axial plies are reasonably close to those for the all-axial case. Since the case of one axial and two circumferential plies would be appropriate (nominally according to netting theory) for a long cylinder pressurized internally, it is fortunate that this case has reasonably high natural frequencies.

\section{EXACT SOLUTION FOR LAMINATED ANISOTROPIC SHELL UNDERGOING AXISYMMETRIC MOTION}

For a shell undergoing axisymmetric motion, the tangential displacement $v$ is identically zero and the axial and normal displacements are independent of tangential position. Then equations (17) can be reduced to 


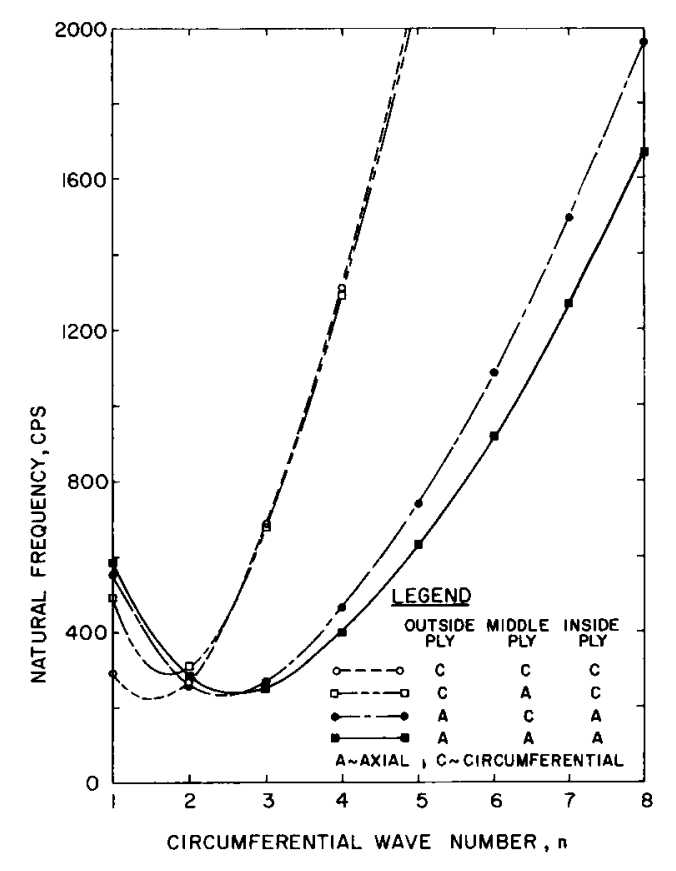

Figure 4. Effect of lamination arrangement on the natural frequencies $(m=1)$ for a three-layer, cross-ply boron/epoxy cylinder.

the following form:

$$
C_{1} w^{\prime V}(x)-2 C_{2} w^{\prime \prime}(x)+C_{3} w(x)=0
$$

where

$$
\begin{aligned}
& C_{1}=\left\{D_{11}+\left(A_{12} B_{11} / \bar{M} \omega^{2}\right)-\left[B_{11}+\left(A_{11} A_{12} / \bar{M} \omega^{2}\right)\right]\left(B_{16} / \bar{A}_{16}\right)\right\} \\
& C_{2}=\left\{B_{12}+\left(A_{12}^{2} / 2 \bar{M} \omega^{2}\right)-\left[B_{11}+\left(A_{11} A_{12} / \bar{M} \omega^{2}\right)\right]\left(\bar{A}_{26} / 2 \bar{A}_{16}\right)\right\} R^{-1} \\
& C_{3}=A_{22} R^{-2}-\bar{M} \omega^{2}
\end{aligned}
$$

It is noted that the coefficients $C_{1}$ and $C_{2}$ contain elastic coefficients with subscripts 16 and 26 not present in the corresponding coefficients for orthotropic or isotropic materials. The explanation for this is that although there are no shear strains produced by the axisymmetric displacement, the normal strains produced by axisymmetric motion induce shear stresses as a result of anisotropic cross-elasticity effects.

The solution of equation (25) can be expressed as follows:

$$
w(x)=\sum_{k=1}^{4} W_{k} e^{\lambda_{k} x}
$$




\section{APPROXIMATE SOLUTION FOR LAMINATED ANISOTROPIC SHELL UNDERGOING UNSYMMETRIC MOTION}

As mentioned before, it is not feasible to expand and solve the characteristic equation in the general anisotropic case. Another approach to obtain an approximate solution is to assume a simplified functional form which satisfies the equations of motion (17) exactly. Due to the presence of terms containing odd mixed partial derivatives, the checkerboard modes expressed by equation (23) do not satisfy equations (17). To accommodate these terms, functions having the combined argument $(\alpha x+\beta y)$ are necessary. Motivated by the work of Pagano, Halpin, and Whitney [19], who demonstrated that axial tension of a general anisotropic cylindrical shell produces helical buckling modal patterns, the following helical-mode displacement functions were investigated:*

$$
\begin{gathered}
u=U \cos (\alpha x+\beta y) \sin \omega t, v=V \cos (\alpha x+\beta y) \sin \omega t \\
w=W \sin (\alpha x+\beta y) \sin \omega t
\end{gathered}
$$

It was found that these functions are exact solutions of the equations of motion**. Unfortunately, since the nodal pattern associated with such a function is helical (see Figure 5), it cannot satisfy any boundary conditions at the ends ( $x=$ constant). However due to the effects of the terms with odd mixed partial derivatives, the natural frequency calculated for a specific $\alpha$ and $\beta$ is different from that calculated for the same value of $\alpha$ and a $\beta$ value equal in magnitude but opposite in sign. Thus, contrary to the case for specially orthotropic or isotropic shells, the $+\beta$ and $-\beta$ solutions are linearly independent solutions. Thus, it was decided to use the following two functions for $w$ (along with analogous ones for $u$ and $v$ ):

$$
w=\left[W_{1} \sin \left(\alpha_{1} x+\beta_{y}\right)+W_{2} \sin \left(\alpha_{2} x-\beta y\right)\right] \sin \omega t
$$

where $W_{1}$ and $W_{2}$ are constants. Following the approach suggested by Nikulin for isotropic shells, the ratio $W_{2} / W_{1}$ is determined so that the primary boundary condition $(w=0)$ is satisfied exactly at each end of the shell. Equation (28) can be expanded as follows:

$$
\begin{aligned}
w=[ & \left(W_{1} \sin \alpha_{1} x+W_{2} \sin \alpha_{2} x\right) \cos \beta y \\
& \left.+\left(W_{1} \cos \alpha_{1} x-W_{2} \cos \alpha_{2} x\right) \sin \beta y\right] \sin \omega t
\end{aligned}
$$

\footnotetext{
- Kunukkasseril [8] demonstrated analytically that such modes occur in cylindrical shells with closely spaced helical stiffeners and Koval and Cranch $[20]$ showed experimentally that application of an initial static torque tended to rotate the axial nodal lines into helices.

ofimilar terms with the trigonometric functions replaced by the corresponding hyperbolic functions of the same argument were also found to satisfy the equations of motion. However, they are not possible solutions for a complete (circumferentially closed) shell, such as the one considered here, since they are not periodic (single-valued) in the circumferential coordinate $y$.
} 


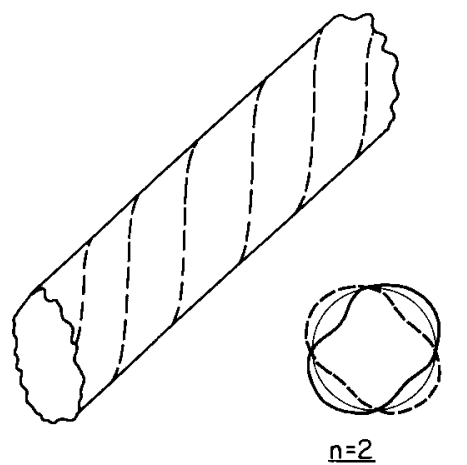

Figure 5. Single helical nodal pattern.

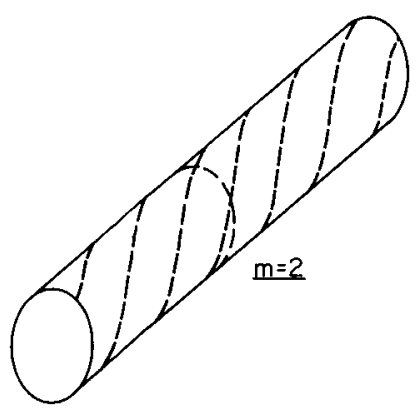

Figure 6. Typical nodal pattern associated with combination of two helical modes.

Now it is convenient to choose the origin of the $x$ axis at the middle of the shell length. Then the primary boundary conditions are:

$$
w( \pm \ell, y)=0
$$

where $\ell=L / 2$.

This results in the following set of homogeneous, linear, algebraic equations in the constants $W_{1}$ and $W_{2}$ :

$$
\left[\begin{array}{rr}
\sin \alpha_{1} \ell & \sin \alpha_{2} \ell \\
\cos \alpha_{1} \ell & -\cos \alpha_{2} \ell
\end{array}\right]\left\{\begin{array}{l}
W_{1} \\
W_{2}
\end{array}\right\}=0
$$

To assure a nontrivial solution of equations (31), the determinant of the coefficients must be equal to zero. This leads to the following transcendental equation:

$$
\cos \alpha_{1} \ell \sin \alpha_{2} \ell+\sin \alpha_{1} \ell \cos \alpha_{2} \ell=0
$$

The solution of equation (32) is expressed simply as follows:

$$
\alpha_{1}+\alpha_{2}=\pi p / \ell
$$

where $p$ is any integer other than 0 . Figure 6 shows a typical nodal pattern.

The procedure used to obtain an approximate solution is as follows. From the frequency determinant obtained from the coupled equations of motion for a single helical mode, frequency is calculated and plotted as a function of $m_{1}$ for the desired value of circumferential wave number $n$. Similarly frequency is determined and plotted versus $m_{2}$, using the same single helical mode but a negative value of $n$. For graphical convenience, equation (33) is rewritten as follows:

$$
\left(m_{1}+m_{2}\right) / 2=p
$$




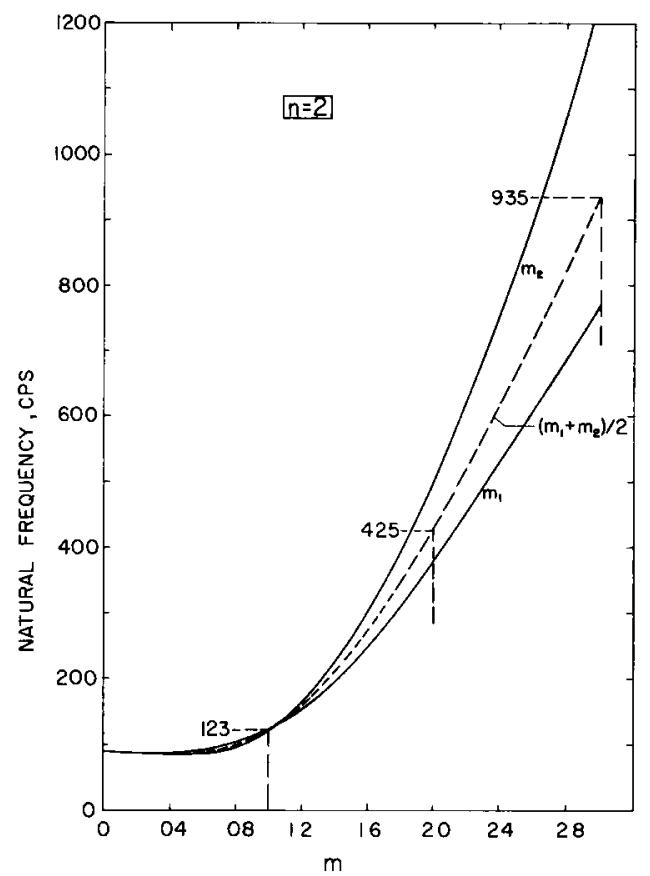

Figure 7. Graphical solution to determine natural frequencies.

Then a third line is drawn in which frequency is plotted against the mean value of $m_{1}$ and $m_{2}$ (shown as a dashed line in Figure 7). Finally, the natural frequencies are those frequencies at which the frequency vs. $\left(m_{1}+m_{2}\right) / 2$ curve intersects integer values of $\left(m_{1}+m_{2}\right) / 2$. Thus, Figure 7 shows that the lowest natural frequency for $n=2$ occurs at approximately $123 \mathrm{cps}$, the second frequency at approximately $425 \mathrm{cps}$, and the third at approximately $925 \mathrm{cps}$. Since the two curves of frequency vs. $m_{1}$ and vs. $m_{2}$ are generally similar, it is generally sufficiently accurate to assume that the frequency associated with $\left(m_{1}+m_{2}\right) / 2$ is approximated with sufficient accuracy by

$$
\omega \approx\left[\omega\left(m_{1}\right)+\omega\left(m_{2}\right)\right] / 2
$$

The shell used in the illustrative example of Figure 7 has the same geometry as the boron/epoxy laminated cylinder considered previously, except here, the outer layer is oriented circumferentially, while the inner layer is oriented at $\mathbf{4 5}$ degrees to exaggerate the tangential anisotropic effect. Figure 8 shows natural frequency associated with $m=1$ as a function of circumferential wave number.

For the same shell geometry and a fixed outer-layer orientation 


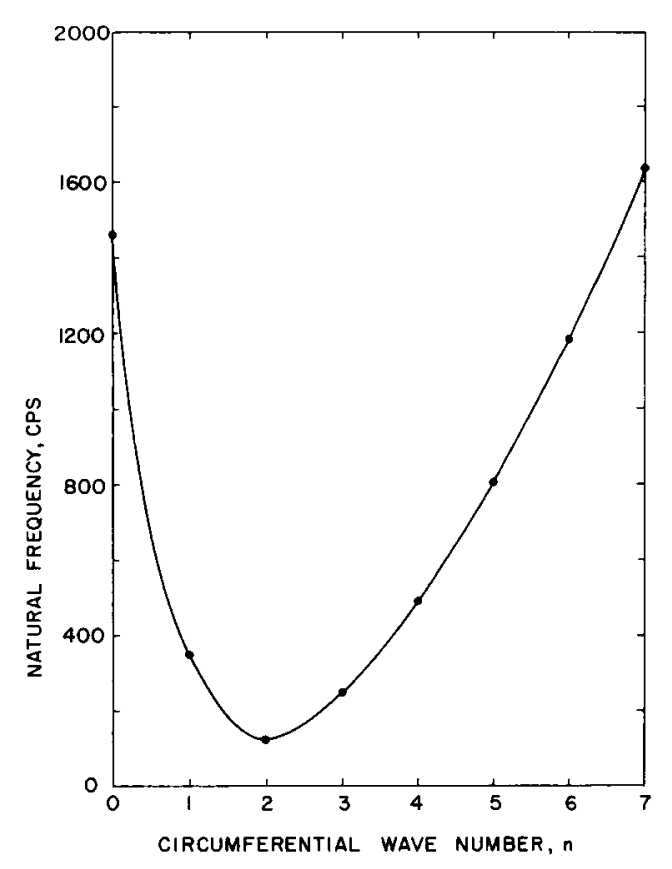

Figure 8. Effect of circumferential wave number on natural frequencies $(m=1)$ of a two-layer boron/epoxy cylinder with outer layer circumferential, inner at 45 degrees.

(circumferential), Figure 9 shows the effect of inner-layer orientation on the frequencies for $m=1$ and various values of $n$. It is interesting to note that the lowest frequency is associated with $n=2$ regardless of inner-layer orientation. Although inner layer orientation has only a weak effect on this lowest frequency, it is maximized by selecting an axial orientation (0 degrees).

\section{CONCLUSIONS}

On the basis of the present investigation, the following conclusions are drawn:

1. The concept of using an equivalent homogeneous isotropic shell, as proposed by Weingarten, to predict the behavior of a shell with multiple isotropic layers, all having the same Poisson's ratio, is valid. Of course, this concept cannot be applied to multiple anisotropic layers.

2. The effect of bending-stretching coupling in an unsymmetrically laminated shell can be appreciable, as demonstrated here for a two-layer 
Free Vibrations of Multilayer Anisotropic Cylindrical Shells

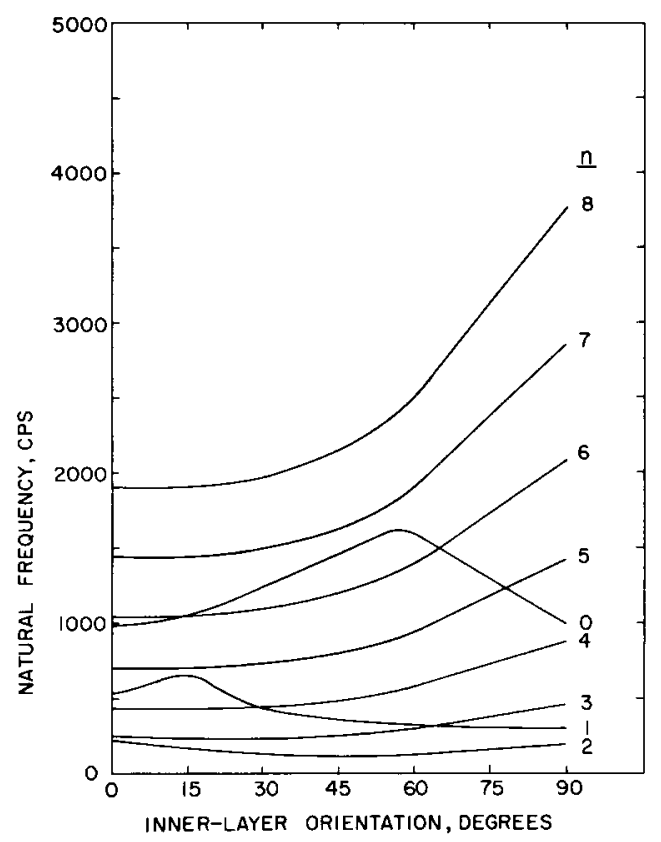

Figure 9. Effect of inner-layer orientation on the natural frequencies $(m=1)$ for a two-layer boron/epoxy cylinder with outer layer circumferential.

boron-epoxy shell.

3. The exact solutions for two helical-type modes can be combined to obtain an approximate solution for a finite-length shell.

\section{ACKNOWLEDGMENT}

The assistance of the University of Oklahoma Computing Center in performing the computations on an IBM System 360 Series 40 is gratefully acknowledged.

\section{NOMENCLATURE}

$A_{i j} \quad=$ elements of stretching stiffness matrix for shell

$B_{i j} \quad=$ elements of bending-stretching coupling matrix for shell

$C_{i} \quad=$ coefficients defined in equations (25)

$D_{i j} \quad=$ elements of bending stiffness matrix for shell

$d_{x}, d_{y}, d_{t}=$ linear partial differential operators with respect to $x, y, t$

$h \quad=$ total shell wall thickness

$i, j \quad=$ indexes $1,2,6$ 


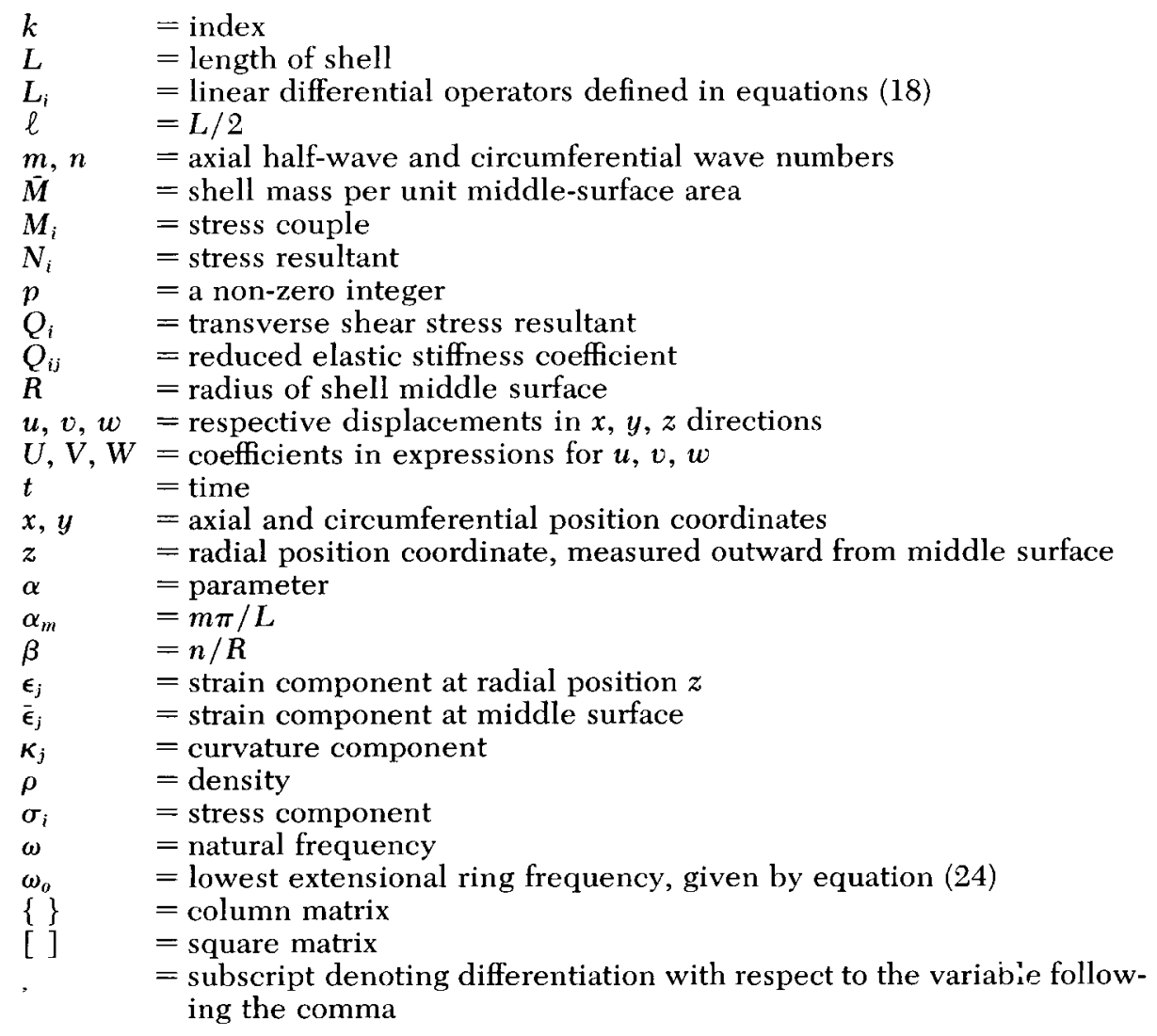

\section{REFERENCES}

1. W. H. Hoppmann, II, "Some Characteristics of the Flexural Vibrations of Orthogonally Stiffened Cylindrical Shells," J. Acoust. Soc. America, Vol. 30 (1958), p. 77.

2. Y. C. Das, "Vibrations of Orthotropic Cylindrical Shells," Applied Scientific Research, Vol. 12A (1964), p. 317.

3. V. I. Weingarten, "Free Vibrations of Multilayered Cylindrical Shells," Experimental Mechanics, Vol. 4 (1964), p. 200.

4. D. Bushnell, "Dynamic Response of Two-Layered Cylindrical Shells to Time-Dependent Loads," AIAA Journal, Vol. 3 (1965), p. 1698.

5. R. M. Jones and S. Klein, "Equivalence Between Single-Layered and Certain Multilayered Shells," AIAA Journal, Vol. 6 (1968), p. 2295.

6. J. C. White, "The Flexural Vibrations of Thin Laminated Cylinders," Jour. of Eng. for Industry, Trans, ASME, Vol. 83B (1961), p. 397.

7. S. B. Dong, "Free Vibrations of Laminated Orthotropic Cylindrical Shells," J. Acoust. Soc. America, Vol. 44 (1968), p. 1628.

8. V. X. Kunukkasseril, "Vibration of Multi-Layered Anisotropic Cylindrical Shells," Report WVT-6717, Watervliet Arsenal (Feb. 1967). AD-649662. 
9. A. E. H. Love, A Treatise on Mathematical Theory of Elasticity, 4th ed., Dover Publications, New York (1944).

10. L. H. Donnell, "Stability of Thin-Walled Tubes Under Torsion," NACA Report 479 (1933).

11. W. Flügge, Statik und Dynamik der Schalen, Springer, Berlin, Germany (1934).

12. S. B. Batdorf, "A Simplified Method of Elastic Stability Analysis of Thin Cylindrical Shells," NACA Report 874 (1947).

13. S. B. Batdorf, "On the Application of Inverse Differential Operators to the Solution of Cylinder Buckling and Other Problems," AIAA/ASME 10th Structures, Structural Dynamics and Materials Conference, Collection of Technical Papers on Structures and Materials, p. 386 (Apr. 1969).

14. M. V. Nikulin, "Free Oscillations of Smooth and Structurally Anisotropic Cylindrical Shells in the Presence of Static Loads," Prochnost' i dinamika aviatsionnykh dvigateley, Sbornik Statey no. 2 (1965), p. 52; English translation, Foreign Technology Division, FTD-MT-66-145 (Dec. 18, 1967), p. 35. AD-675270.

15. K. Forsberg, "Influence of Boundary Conditions on the Modal Characteristics of Thin Cylindrical Shells," AIAA Journal, Vol. 2 (1964), p. 2150.

16. D. M. Egle and F. M. Bray, "An Experimental Study of the Free Vibration of Cylindrical Shells with Discrete Longitudinal Stiffening," School of Aerospace and Mechanical Engineering, University of Oklahoma, Final Report, National Science Foundation Grant GK-1490 (Nov. 1968).

17. D. M. Egle and K. E. Soder, Jr., "A Theoretical Analysis of the Free Vibration of Discretely Stiffened Cylindrical Shells with Arbitrary End Conditions," School of Aerospace and Mechanical Engineering, University of Oklahoma, NASA Grant NGR 37-003-035, Final Report, Part I (Aug. 1968); also AIAA Symposium on Structural Dynamics and Aeroelasticity, New Orleans, Louisiana, p. 262 (Apr. 1969).

18. M. E. Waddoups, "Characterization and Design of Composite Materials," Composite Materials Workshop, S. W. Tsai, J. C. Halpin, and N. J. Pagano, editors, Technomic Publishing Co., Inc., Stamford, Conn., p. 254 (1968).

19. N. J. Pagano, J. C. Halpin, and J. M. Whitney, "Tension Buckling of Anisotropic Cylinders," J. Composite Materials, Vol. 2 (1968), p. 154.

20. L. R. Koval and E. T. Cranch, "On the Vibrations of Thin Cylindrical Shells Subjected to an Initial Static Torque," Proc. of the 4th U. S. National Congress of Applied Mechanics, ASME, Vol. 1 (1962), p. 107. 